\title{
Metal ion reductions by femtosecond laser pulses with micro-Joule energy and their efficiencies
}

\author{
Nobuaki Nakashima, Ken-ichi Yamanaka, Morihisa Saeki, \\ Hironori Ohba, Seiji Taniguchi, Tomoyuki Yatsuhashi
}

\begin{tabular}{|c|l|}
\hline Citation & Journal of Photochemistry and Photobiology A: Chemistry. 319-320; 70-77 \\
\hline Issue Date & $2016-03-15 \_2016-04-15$ \\
\hline \multicolumn{1}{|c|}{ Type } & Journal Article \\
\hline Textversion & author \\
\hline Highlights & $\begin{array}{l}\text {-This paper describes a part of primary mechanisms of metal ion reductions leading } \\
\text { to metal nano-particle (NP) formation by femtosecond laser pulses, even in the laser } \\
\text { intensity range of micro-Joule/pulse, where the intensities were around the } \\
\text { supercontinuum (SC) generation threshold. } \\
\text {-The new findings are the reduction efficiencies of the metal ions } 10^{-3} \text { per incident } \\
\text { photon, and major absorbing species of the laser energy was water. } \\
\text { ·We estimate that the solvated electrons act as a reducing agent and that direct } \\
\text { multi-photon dissociation to neutral atoms was unlikely. }\end{array}$ \\
\hline Rights & $\begin{array}{l}\text { C2016 Elsevier B.V. This manuscript version is made available under the } \\
\text { CC-BY-NC-ND 4.0 License. http://creativecommons.org/licenses/by-nc-nd/4.0/. } \\
\text { The article has been published in final form at } \\
\text { https://doi.org/10.1016/j.jphotochem.2015.12.021 }\end{array}$ \\
\hline DOI & \begin{tabular}{l}
$10.1016 /$ j.jphotochem.2015.12.021 \\
\hline
\end{tabular} \\
\hline
\end{tabular}

Self-Archiving by Author(s) Placed on: Osaka City University Repository 


\title{
Metal ion reductions by femtosecond laser pulses with micro- Joule energy and their efficiencies
}

\author{
Nobuaki Nakashima, ${ }^{* 1}$ Ken-ichi Yamanaka, ${ }^{2}$ Morihisa Saeki, ${ }^{3}$ Hironori Ohba, ${ }^{3}$ Seiji \\ Taniguchi, ${ }^{4}$ and Tomoyuki Yatsuhashi ${ }^{1}$ \\ ${ }^{1}$ Department of Chemistry, Graduate School of Science, Osaka City University, \\ Sumiyoshi, Osaka 558-8585 \\ ${ }^{2}$ Toyota Central R\&D Labs., Inc., Nagakute, Aichi 480-1192 \\ ${ }^{3}$ Quantum Beam Science Directorate, Japan Atomic Energy Agency, Tokai-mura, Naka- \\ gun, Ibaraki 319-1195 \\ ${ }^{4}$ Institute for Laser Technology, Osaka, 550-0004 \\ E-mail: <nakashima@toyotariken.jp>
}




\section{ABSTRACT}

Chemical reactions of metal ions in solution were studied using $800 \mathrm{~nm}, 90$ femtosecond laser pulses with a laser intensity range beginning at sub-micro-Joules/pulse, where the intensities were at or just below supercontinuum generation. Products were observed as surface resonance absorptions assignable to Ag, Au nanoparticles (NPs) and light scattering, indicating the formation of Pd NPs. The consumption efficiencies of metal ions from the $\mathrm{Au}$ and $\mathrm{Pd}$ ions were estimated to be $10^{-3}$ per incident photon. The major absorbing species of the laser energy was water, which led to the formation of solvated electrons that acted as a reducing agent of metal ions, while direct multi-photon dissociation to neutral atoms was unlikely. Metal ion $\mathrm{Fe}^{3+}$ and $\mathrm{Yb}^{3+}$ systems are also discussed, where $\mathrm{Fe}^{3+}$ to $\mathrm{Fe}^{2+}$ was ascribed to two-photon absorption and $\mathrm{Yb}^{3+}$ to $\mathrm{Yb}^{2+}$ to a reduction followed by solvent ionization. 


\section{Introduction}

Metal ion in solutions can be reduced by focused femtosecond laser pulses. This method was successfully used for the reduction of $\mathrm{Eu}^{3+}$ to $\mathrm{Eu}^{2+}$ in methanol in 2008.[1] In our previous reports, three $\mathrm{M}^{3+}(\mathrm{M}=\mathrm{Sm}, \mathrm{Yb}, \mathrm{Fe})$ ions were changed to their $\mathrm{M}^{2+}$ counterparts.[2-3] The $\mathrm{Eu}^{3+}, \mathrm{Sm}^{3+}, \mathrm{Yb}^{3+}$ ions are suggested to be reduced by electrons produced from water ionization by focused femtosecond pulses. The mechanisms of $\mathrm{Fe}^{3+}$ to $\mathrm{Fe}^{2+}$ depended on excitation wavelengths; the same as the above at $1.2 \mu \mathrm{m}$ and twophoton chemistry at $800 \mathrm{~nm}$. [2-3] In the case of singly and/or doubly charged ions, they seem to develop into nanoparticles (NPs). NP formation using femtosecond pulses has been extensively studied, including for NPs of $\mathrm{Au}[4-9], \mathrm{Ag}[5,10,11], \mathrm{Pd}[12,13]$, and others.[14] The NP formation would be initiated by a reduction of charged metal and complex ions to neutral atoms by electrons produced by water ionization and/or direct multi-photon atomization.[8] These reactions are often accompanied by supercontinuum (SC) generation.[1, 3, 9, 11] The $\mathrm{M}^{3+}$ ion reductions and NP formation would have the common initial mechanisms.

These metal ion reductions have raised several important questions that remain to be clarified. i) First, what are the major absorbing species in the sample? Because these reductions are accomplished using focused femtosecond laser pulses, water is presumably ionized by a five-photon absorption. If so, ionic species and surfactants (additives) might be a source of electrons and atoms via low order, 2-4 photon absorptions. The simple absorption experiments in this paper reveal that water is the major absorption species and atomization and/or surfactant ionization via multi-photon absorption makes a minor contribution. 
ii) Second, how efficient are these reductions? In the present study, we successfully estimated the initial reduction efficiencies for the formation of NPs based on the spectral changes of metal ions. We found that the efficiencies were relatively low, which leads to the following question.

iii) Third, what are the competing reactions for the initial reductions? We consider that electron quenching reactions by the electrons themselves and/or $\mathrm{OH} \bullet$ radicals are the most probable candidates. High electron density states would be generated, leading to efficient electron quenching reactions and, as a result, low reduction efficiencies. These high electron density states - i.e., weak plasma states — would be generated in a small volume when using focused femtosecond pulses due to water absorption/ionization, accompanied by SC generation.[15, 16]

To resolve these questions and clarify the underlying mechanisms, a low energy femtosecond laser pulse would be preferable. In this paper, the formation of NPs was observed using pulses in the energy range of micro-Joules, which to our knowledge is the lowest range shown to form NPs, and is the same energy range at which $\mathrm{M}^{3+}$ reduction occurs accompanied by SC generation.[3] In previous reports, formation of NPs was accomplished using pulses with rather high energy of $0.3-0.5$ milli-Joules/pulse with a duration of 30-130 fs.[4, 6, 7, 9-14] This sub-milli-Joule laser irradiation condition is known as the strong optical breakdown (SOB) condition.[5] An optical breakdown (OB) of the medium generates vigorous plasma conditions with bubble formation. High laser intensity conditions of SOB would involve many active species. [17] Low-opticalbreakdown (LOB) conditions would have been recommended.[5] In another study performed for the purpose under LOB conditions, femtosecond pulses were elongated to 
36 ps to suppress peak intensities.[8] In this paper, we observed the formation of NPs by measuring the absorption and scattering of the sample solutions, and estimated the consumption efficiencies. Finally, based on our results, we discuss the reduction mechanisms in the region of LOB conditions. 


\section{Experimental}

The sample solutions had a volume of $0.4 \mathrm{~cm}^{3}$ in a 5 (width) $\times 10$ (depth) $\mathrm{mm}^{2}$ glass cell. The femtosecond laser beam was introduced in the direction of $10 \mathrm{~mm} \mathrm{depth}$, and absorption of the solution was measured with a path length of $10 \mathrm{~mm}$ after typically 30 min irradiation. The laser with a mode-locked Ti:sapphire oscillator, and the pulse shape and power measurement systems were the same as those used previously.[18] The output of the amplifier (1.9 W, 90-100 fs fwhm, $1 \mathrm{kHz}, 800 \mathrm{~nm}, 1 / \mathrm{e}^{2}$ width of $12 \mathrm{~mm}$ ) was reflected by wedged plates and thereby weakened to a micro-Joules $(\mu \mathrm{J}) /$ pulse level. A plano-convex lens with a focal length of $230 \mathrm{~mm}$ was used to focus the laser beam into a sample cell at the focal point. The averaged power of the laser pulses was monitored with power meters with an aperture size of $19 \mathrm{~mm}$ (Gentec TPM310). Some of the transmission measurements were also done by using this thermocouple-type power meter. SC spectra were measured with an Ocean Photonics USB4000 spectrometer.

Using a second femtosecond laser system, major transmission measurements were done for water and metal ion solutions using quartz cells through an integrated sphere with an aperture size of $20 \mathrm{~mm}$ (Hamamatsu A10094-03) attached to a Ge detector THORLABS PDA 50B-EC and/or a Si detector THORLABS DET100A/M. As a second femtosecond laser system, a Ti:sapphire laser (Coherent: Legend Elite USP-HE 30-35 fs, $5 \mathrm{kHz}, 0.8 \mathrm{~mJ} /$ pulse, $1 / \mathrm{e}^{2}$ width of $6.2 \mathrm{~mm}$ ) was used.[19] The laser beam was reflected by wedged plates and the weakened pulse was directed toward the focusing lens. A mechanical shutter was placed before the focusing lens and gave a trapezoidal open time (a few $\mu$ s) consisting of 13 laser pulses of equal intensity. One of them was picked up to measure the pulse intensity. Without the mechanical shutter, the intensities fluctuated at times, presumably because the laser light was scattered by the previously formed particles. 
Treatment of reflection losses of a sample is noted in the text.

The product NPs were detected by their absorption or scattering spectra, with a peak at $405 \mathrm{~nm}$ for silver,[5, 20-23] $532 \mathrm{~nm}$ for gold, [4, 5, 8, 24] and $700 \mathrm{~nm}[12,13,25-$ 27] for palladium NPs using a Shimadzu UV 3600 spectrophotometer. NPs of Ag and Au were confirmed using a JEM-2100F transmission electron microscope (TEM) from JEOL. Sodium dodecyl sulfate (SDS, 0.01M) and 0.5 M 2-propanol were added to $0.05 \mathrm{M}$ $\mathrm{AgClO}_{4}$ solution. Solutions of $\mathrm{HAuCl}_{4} \cdot 4 \mathrm{H}_{2} \mathrm{O} 0.5 \mathrm{mM}$ with $0.01 \mathrm{M} \mathrm{SDS}$ in $\mathrm{H}_{2} \mathrm{O}$ were used. $\mathrm{PdCl}_{2} 2.75 \mathrm{mM}$ with $0.055 \mathrm{M} \mathrm{HCl}$ in a 3:1 solution of water: ethanol were irradiated without SDS. For $\quad$ Fe-complex experiments, $0.1 \quad \mathrm{M}$ potassium tris(oxalate)ferrate(III)trihydrate $\left(\mathrm{K}_{3} \mathrm{Fe}_{2}\left(\mathrm{C}_{2} \mathrm{O}_{4}\right)_{3} \cdot 3 \mathrm{H}_{2} \mathrm{O}\right)$ (Wako Chemicals) in acidic solution was used. 


\section{Results}

3.1 Water as a major absorbing species for focused laser pulses and supercontinuum generation

Figures 1 a) and b) show transmission arrangements for the absorption measurements. When a sample was located at a defocused point as shown Fig. 1 a), the ratio of output laser intensity $I_{0}$ vs. input intensity $I_{0}{ }^{0}, T_{0}{ }^{0}=I_{0} / I_{0}{ }^{0}$, was 0.87 for an empty $1-\mathrm{cm}$ long cell and 0.91 for a water-filled sample. These values are in agreement with the observed ones by a spectrophotometer at $800 \mathrm{~nm}$ within 0.005 errors. When a water-filled sample was set at the focal point at $230 \mathrm{~mm}$ as shown Fig. $1 \mathrm{~b}$ ), the transmission light intensity $I$ was further decreased. The additional absorption was evaluated by the transmission of $T=I / I_{0}$ (water-filled sample). The typical $T$ value was 0.83 for a 0.9 $\mu \mathrm{J} /$ pulse of the second 30-35-fs-laser system measured by an integrated sphere with a PD. The $T$ value of 0.85 was also observed for a $2.9 \mu \mathrm{J} /$ pulse of the first 90 -fs-laser system measured by a thermocouple detector, with an estimated error of 0.05 . About $15 \%$ of the input energies were additionally absorbed by water by placing the sample at a focal point. It is notable that there was no effect of diffraction changes by the nonlinear index in the sample on the detector, because the detectors have large apertures of $20 \mathrm{~mm}$ for the integrated sphere and $19 \mathrm{~mm}$ for the thermocouple. 
a)

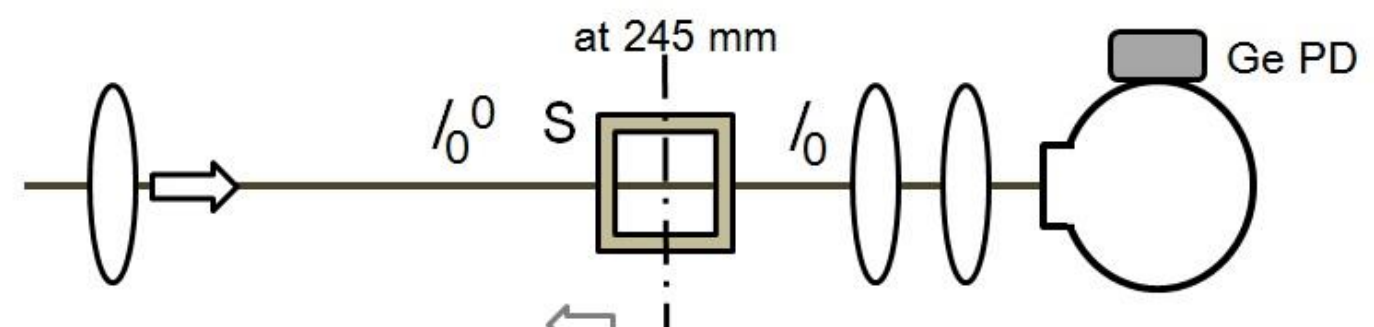

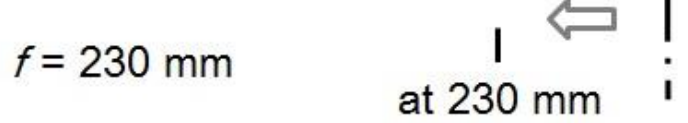

b)

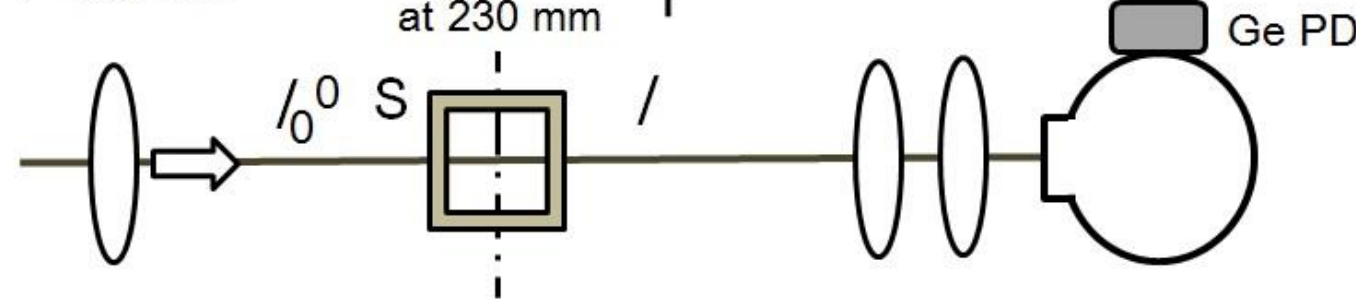

c)

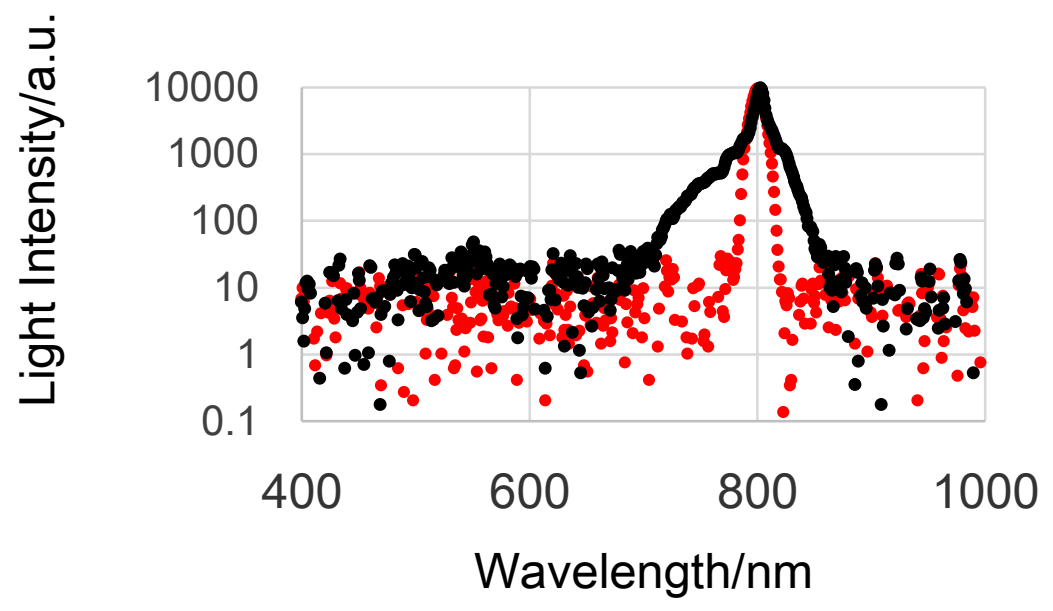

Figures 1 a) and b) show the optical arrangements for transmission. a) The sample was placed at a defocused position of $245 \mathrm{~mm}, 15 \mathrm{~mm}$ shifted from the focusing point of 230 $\mathrm{mm}$. b) The sample was placed at the focal point of a lens with a $230 \mathrm{~mm}$ focal length. The transmitted pulse intensities $I_{0}$ from a) and $I$ from b) were measured by a PD after an integrated sphere. c) Laser spectra from a 1-cm-water-filled sample at a position a) (red) and b) (black). 
The $T$ values can be compared between water itself and sample solutions under the same detection system. The $T$ value for water was $0.83 \pm 0.01$ and the same values were observed for the Ag and Au salt solutions, which contained ionic salts and additives of SDS $10^{-2} \mathrm{M}$. A SDS $10^{-2} \mathrm{M}$ solution also showed the same $T$ value. The $T$ value measurements led to an important conclusion: Water is the major absorbing species and the absorption amount was ca. 15\%; no appreciable extra absorptions were observed for the Ag and Au salts solutions used for the present experiments. Yellow colored solutions of Pd and Fe salts showed a difference. They absorbed laser energy more effectively, with $T$ values of 0.80 for the $\mathrm{Pd}$ and 0.75 for the Fe system. The almost same results were obtained for a $2.9 \mu \mathrm{J} /$ pulse of the 90 -fs-laser system measured by a thermocouple detector.

$\mathrm{SC}$ was observed under the above irradiation conditions, as shown in Fig. 1 c) at $3 \mu \mathrm{J}$ with a 90 -fs pulse irradiation with and without a $1 \mathrm{~cm}$ water sample. The width of the laser spectrum at $1 \%$ of the peak intensity was $31 \mathrm{~nm}$ and a broadening was seen to $125 \mathrm{~nm}$ with water. Asymmetric and anti-storks broadening characteristics in the SC spectrum were clearly seen. The component of the SC in the 400-600 nm region was just discernible above the background $\left(10^{-3}\right.$ of the top); in fact, the SC was visible as a stable white light. The SC from water has been studied in detail.[16] and the resulting spectra provide a good standard. We realize that the SC spectra in Fig.1 c) indicate that the focused laser pulses created a weak plasma state and can be classified into the region above the SC threshold, based on the reference,[16] In that investigation, a laser of 170 fs fwhm, $800 \mathrm{~nm}, 1 / \mathrm{e}^{2}$ width of $4 \mathrm{~mm}$ and focusing lens of $11.9 \mathrm{~cm}$ were used to measure SC to a low background $\left(<10^{-6}\right.$ of the top) level. The width of the laser spectrum at $1 \%$ of the peak was $40 \mathrm{~nm}$ and broadening was seen to $80 \mathrm{~nm}$ with water at $2 \mu \mathrm{J}$. A 6 -fold broadening from $3000 \mathrm{~cm}^{-1}$ to $1.8 \times 10^{4} \mathrm{~cm}^{-1}$ occurred in a small energy range from 1.8 to 
$2.2 \mu \mathrm{J}$ pulse.[16] The turning energy at $2 \mu \mathrm{J}$ under the conditions used in this previous study may be called a threshold. This SC behavior has been explained in terms of the mechanism shift to plasma (electron) broadening from molecular $n_{2}$ broadening, where $n_{2}$ is a nonlinear index.

\subsection{Electron formation supported by absorption of femtosecond pulses and} supercontinuum generation

The absorption measurements and SC generations safely predicted that weak plasma states were built up with a high laser intensity. We use values of the electron density of $2 \times 10^{19} \mathrm{~cm}^{-3}$ and a laser intensity of $2 \times 10^{13} \mathrm{~W} / \mathrm{cm}^{2}$ in this paper. Intensity evaluation based on geometrically focused conditions would not be applicable, because the laser beam would have been deformed by the non-linear index $n_{2}$. The adopted values are based on the paper by Milián et al., [15] who observed 10-40\% absorption and provided a detailed analysis with an energy of $0.5-5 \mu \mathrm{J}$ of Ti sapphire laser with a $45-\mathrm{fs}$ fwhm duration, a beam diameter of $7 \mathrm{~mm}$ fwhm (or estimated $1 / \mathrm{e}^{2} 12 \mathrm{~mm}$ ) and focus length of $8.64 \mathrm{~cm}$. The absorption energies are converted a weak plasma state. The electron density can be read as $2 \times 10^{19} \mathrm{~cm}^{-3}$ in $0.1 \mathrm{~mm}$ length from their figures with a resulting laser intensity of $2 \times 10^{13} \mathrm{~W} / \mathrm{cm}^{2}$.[15]

Another support for existing a certain amount density of electrons is SC generation. The SC spectrum of anti-Stokes broadening or blue-shift indicates the interaction of the light with quasi-free electrons.[16] The interaction of the light with the bound electrons of the material can cause only a small, symmetric broadening and no SC. A stable SC in these experiments was observed at $3 \mu \mathrm{J}$ as the spectra in Fig. 1c, was 
observed sporadically at $1.5-2 \mu \mathrm{J}$, and was never observed visually at $0.4-0.65 \mu \mathrm{J}$. At 3 $\mu \mathrm{J}$ irradiation, a certain amount of plasma has already been generated based on the antiStokes asymmetric broadening characteristics in Fig. $1 \mathrm{c}$ ). In all $\mathrm{Ag}, \mathrm{Au}$, and $\mathrm{Pd}$ as well as Fe systems, SC was observed visually, at $3 \mu \mathrm{J}$ pulses. The SC was seen when the laser beam was focused in the water sample according to the arrangement shown in Fig.1 b). It is noteworthy that a new, differently colored SC was seen when the cell positions were changed $\pm 5 \mathrm{~mm}$; the new SC was assignable to the cell window, but of course, there was no SC in the out-of-focus position as in Fig. 1 a).

\subsection{Nano-Particle formation based on spectral changes}

Metal ion systems give spectral changes as shown in Figs. 2 and 4 with a laser energy from 0.65 to $3.1 \mu \mathrm{J} /$ pulse, $15-120 \mathrm{~min}$ at $3 \mu \mathrm{J} /$ pulse, and $90 \mathrm{~min}$ at $6 \mu \mathrm{J} /$ pulse. The characteristic absorption with a peak at $405 \mathrm{~nm}$ for the $\mathrm{Ag}$ system is known as $\mathrm{Ag}$ surface plasmon resonance (SPR) absorption; [5, 20-23] therefore, these spectral changes are assignable to Ag NP formation. No significant spectral shift was observed after a few hours. The NPs had a size of about $20 \mathrm{~nm}$ by electron microscopy.

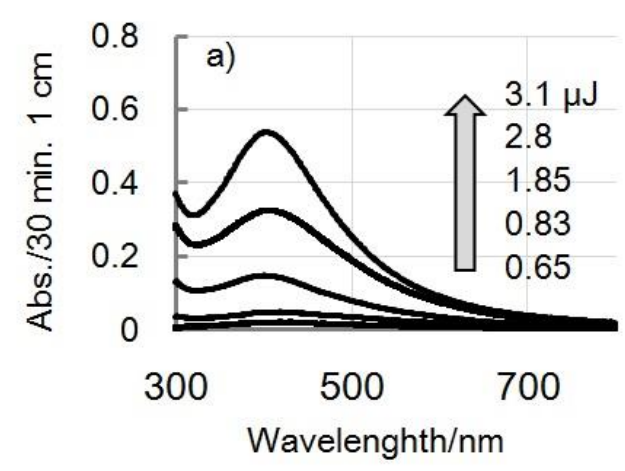




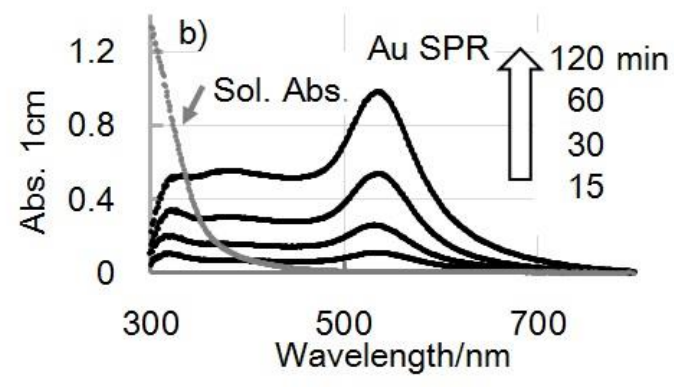

Figures 2 a) Absorption changes of Ag NPs formed by irradiating an $\mathrm{Ag}^{+}$system of 0.05 $\mathrm{M}$ of $\mathrm{AgClO}_{4}$ with $0.01 \mathrm{M}$ SDS in $\mathrm{H}_{2} \mathrm{O}$ with 30 -min irradiation at $1 \mathrm{kHz}$, with the pulse energies in units of micro Joules/pulse as indicated. b) Absorption changes of Au NPs formed by irradiating an aqueous solution of $\mathrm{HAuCl}_{4} \cdot 4 \mathrm{H}_{2} \mathrm{O} 0.5 \mathrm{mM}$ with $0.01 \mathrm{M}$ SDS in $\mathrm{H}_{2} \mathrm{O}$, with the pulse energies at $3 \mu \mathrm{J} /$ pulse for the indicated amounts of time. The left gray line is the absorption spectrum of the original salt solution.

Absorption changes in the Au system, i.e., difference spectra from the original gray color spectrum are shown in Fig. 2 b). The solution color turned wine red and the spectral shape with the peak at $532 \mathrm{~nm}$ was ascribed to the SPR absorption of Au NPs.[4, 5, 8, 24] Suppression of the absorption intensity was seen in the short-wavelength region at 120 min, and could be explained in terms of the consumption of $\mathrm{HAuCl}_{4}$ in a fresh sample. The amount of suppression was used to estimate the reaction efficiency, as discussed later. The absorption intensities increased almost linearly with time. This was also true for the case of Ag NPs if the absorbance value was lower than 0.5 . The spectral peaks remained almost constant, while the peak shifts were observed in SPR absorption of Au NPs under high laser intensity conditions.[6, 9, 28] At a few days after irradiation, the color faded away and the electron microscope images showed that the Au NPs were about $100 \mathrm{~nm}$ in 
size and composed of an aggregate of small (about 10-nm) NPs.

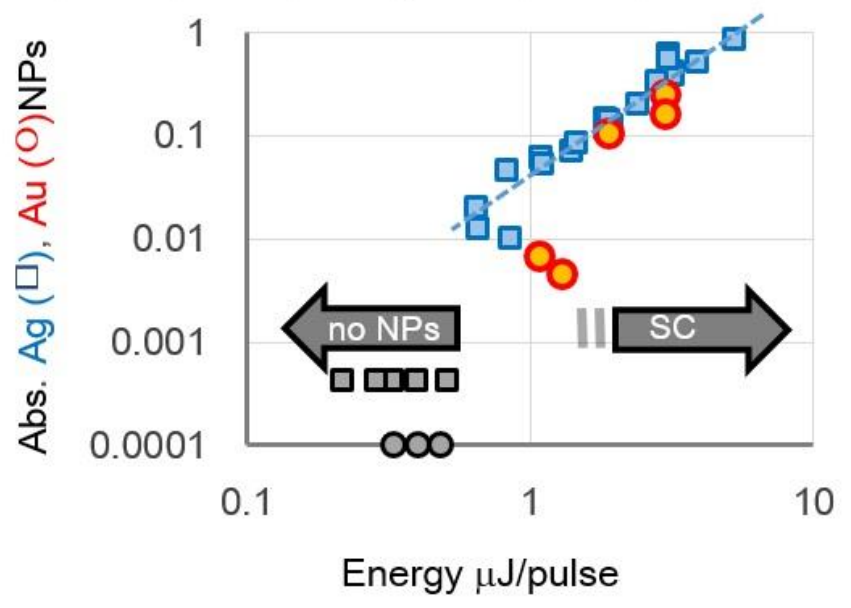

Figure 3 Absorbance values for Ag (squares, $405 \mathrm{~nm}$ ) and Au (circles, $532 \mathrm{~nm}$ ) NPs were plotted versus the laser pulse energy in both logarithmic scales. The dotted guide line represents the absorbance values for Ag NPs.

The peak absorbance values for Ag and Au NPs are plotted in Fig. 3 versus the input laser energy. The Ag NP absorption appeared from a laser intensity of even less than $\mu \mathrm{J} /$ pulse, as shown by the blue squares. A single straight line for the energy dependence could not be drawn down to a region with a low intensity of sub- $\mu \mathrm{J} /$ pulse. A sudden drop resembling threshold behavior was seen in the energy range of sub- $\mu \mathrm{J} /$ pulse, where absorbance values below the present detection limit are denoted by gray squares. The region at left with a gray arrow in Fig. 3 is a region of no NP formation with gray symbols with errors of 0.001 in absorbance units. The area on the right with an SC arrow shows the region of the stable SC at a pulse energy higher than $3 \mu \mathrm{J} /$ pulse accompanied by NPs. Sporadic SC was seen in the region with two bars and no visual SC in the further low intensity region. Spark emissions due to OB were visually seen at times in the region of 
ca. $10 \mu \mathrm{J} /$ pulse. These SC and OB characteristics were not substantially different from those in the previous detailed studies, at least in the case of the spectra in Fig. 1c and the input energy dependency.[16, 29] The SPR absorption values for Au NPs, indicated by the red circles in Fig. 3, were observed within the energy region as the SPR absorptions for the Ag NPs. SC generation in a range of $3 \mu \mathrm{J} /$ pulse indicates electrons had already generated; therefore, it is reasonable that metal ion reductions leading to NPs occurred. On the other hand, the formation of Ag NPs at a laser energy lower than $1 \mu \mathrm{J}$ with a duration of 90 fs suggests that $\mathrm{e}_{\mathrm{sol}}{ }^{-}$had generated. This is simply due to the SC being too weak to be seen visually or to be detected by the present sensitivity. Comments on the slope of $n$ are given in section 4.3 of Discussion.

As shown in Fig. 4, femtosecond pulse irradiation was able to induce an absorption spectral change indicating Pd NP formation at a pulse energy of $6 \mu \mathrm{J} /$ pulse. Previously, to form Pd NPs with femtosecond laser irradiation, $[12,13]$ laser pulses with an energy of $300 \mu \mathrm{J} /$ pulse or higher with a duration of 120 -fs were focused by a $200-\mathrm{mm}$-focal-length lens in ethanol. The spectral change in this paper was essentially the same as those observed in the formation of Pd NPs by femtosecond and nanosecond laser irradiations.[12, 13, 25-27] The pale yellow solution turned to a solution with a dark spot at the laser focal point. The absorption values with a peak at $440 \mathrm{~nm}$ were kept constant in the figure and the light scattered by the particles increased the absorption intensities at all wavelengths other than around the absorption peak. 


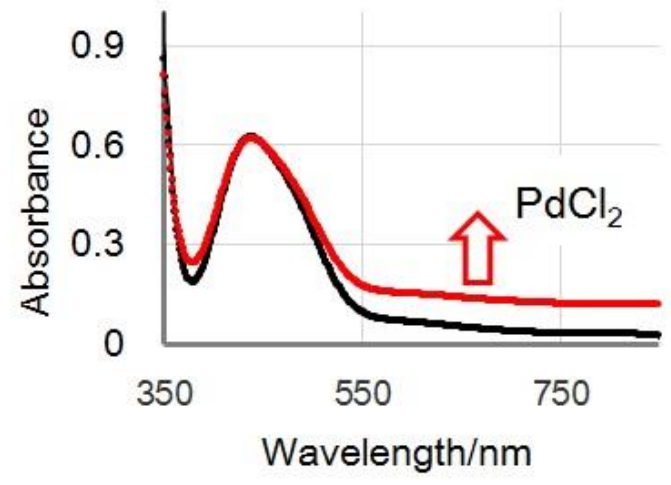

Figure 4 Absorption spectral change by the formation of Pd NPs for a $1 \mathrm{~cm}$ path length at $90 \mathrm{~min}$ irradiation, at a pulse energy of $6 \mu \mathrm{J} /$ pulse. A sample solution with $2.75 \mathrm{mM} \mathrm{PdCl}_{2}$ in $3 / 1\left(\mathrm{H}_{2} \mathrm{O} / \mathrm{EtOH}\right)$ with $0.055 \mathrm{M} \mathrm{HCl}$ was irradiated.

\subsection{Consumption efficiencies of metal ions}

The consumption efficiencies of $\mathrm{Au}$ complex ions and $\mathrm{Pd}^{2+}$ ions are shown in Table 1 with conversion efficiencies for $\mathrm{Yb}^{3+}$ and Fe systems. They were on the order of $10^{-3}$ for the cases considered to involve the solvent ionization mechanism, where reactions with $\mathrm{e}_{\mathrm{aq}}{ }^{-}$would be a common primary mechanism. 
Table 1. Consumption/conversion efficiencies $(\eta)$ of metal ions by focused micro-Joule femtosecond pulses

\begin{tabular}{|c|c|c|c|c|c|}
\hline Salt ${ }^{\text {a) }}$ & Products & $/ \mu \mathbf{J}^{b)}(\mathbf{W L} / \mu \mathrm{m})^{\mathrm{c})}$ & $\eta / \times 10^{-3}$ & Mech. d) & Ref. \\
\hline $\mathrm{HAuCl}_{4}$ & $\mathrm{Au} \mathrm{NP}$ & $3(0.8)$ & 0.7 & SI & This \\
\hline $\mathbf{P d C l}_{2}$ & $\mathrm{Pd} \mathrm{NP}$ & $6(0.8)$ & 0.7 & SI & This \\
\hline $\mathrm{YbCl}_{3}$ & $\mathrm{Yb}^{2+}$ & $10(0.8)$ & 0.8 & SI & $\mathrm{e}$ \\
\hline $\mathrm{K}_{3} \mathrm{Fe}\left(\mathrm{C}_{2} \mathrm{O}_{4}\right)_{3}$ & $\mathrm{Fe}^{2+}$ & $10(0.8)$ & $5 \times 10$ & $\mathrm{TP}$ & $\mathrm{e}$ \\
\hline $\mathrm{K}_{3} \mathrm{Fe}\left(\mathrm{C}_{2} \mathrm{O}_{4}\right)_{3}$ & $\mathrm{Fe}^{2+}$ & $10(1.2)$ & 2 & SI & $\mathrm{e}$ \\
\hline
\end{tabular}

a) Solvents and additives were as described in the text. b) Input pulse energy. c) Laser wavelength 0.8 or $1.2 \mu \mathrm{m}$. d) Mechanisms SI: solvent ionization; TP: two photon absorption. e) Ref.[3]. 
The consumption of metal ions was evaluated from the absorption spectra in Figs. 4 and 5. The Au NP SPR absorption spectrum at $120 \mathrm{~min}$ in Fig. 5, i.e., the top spectrum, is distorted in the UV region by consumption of the original absorption spectrum.

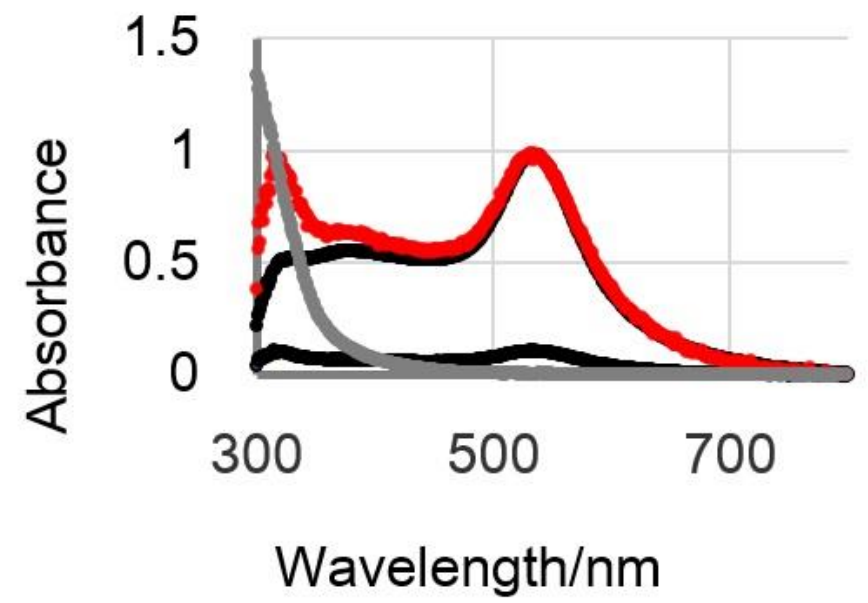

Figure 5 Method for estimating the consumption of Au ions due to the formation of NPs. Au NPs absorptions are shown after irradiation for $15 \mathrm{~min}$ (bottom) and $120 \mathrm{~min}$ (middle black) from Fig. 2b). The orange monotonous spectra in the UV region (gray) are the absorption spectra of the sample before irradiation (the reference solution for two black spectra). The $15 \mathrm{~min}$ spectrum is normalized to the $120 \mathrm{~min}$ one around $530 \mathrm{~nm}$ and longer as the red spectra. The difference between red and black can be attributed to the consumption of the Au ions in the original (orange) solution.

The distortion is ascribed to the consumption of the metal complex ions, as shown in Fig. 5. The consumption of $\mathrm{AuCl}_{4}{ }^{-}$is estimated to be $50 \%$, or $0.25 \mathrm{mM}$, based on the distortion or decrease at $310 \mathrm{~nm}$ from the original $\mathrm{Au}$ ion solution in Fig. 5. The consumption efficiency $\eta$ of $0.7 \times 10^{-3}$ was calculated as follows. The disappeared Au complex ions of $1.0 \times 10^{-7} \mathrm{~mol}\left(0.25 \mathrm{mM}\right.$ of $\left.0.4 \mathrm{~cm}^{3}\right)$ are divided by the input photon mol (Einstein) of $1.44 \times 10^{-4}$. The input laser energy was $21.6 \mathrm{~J}$ by $3 \mu \mathrm{J} /$ pulse for $120 \mathrm{~min}$ at 1 
$\mathrm{kHz}$, at a photon mol corresponding to $1.44 \times 10^{-4}$ Einstein.

For the Pd NPs, the efficiency was $0.7 \times 10^{-3}$ using the spectra in Fig. 4. The consumption of $\mathrm{Pd}$ ions was estimated to be 0.09 in absorbance, corresponding to $3.9 \times 10^{-}$ ${ }^{4} \mathrm{M}\left(1.57 \times 10^{-8} \mathrm{~mol}\right)$ at the absorption of Pd ions around $440 \mathrm{~nm}$, where the absorption values were cancelled by the scattered intensity of the Pd NP. The consumption mol $1.57 \times 10^{-7} \mathrm{~mol}$ was $2.16 \times 10^{-4}$ Einstein $(6 \mu \mathrm{J} /$ pulse for $90 \mathrm{~min})$, yielding an efficiency of $0.7 \times 10^{-3}$. The $\eta$ values have in one significant figure; therefore, the surface reflection losses (about 4\%) were not included in the above calculations. It may be noted that the efficiency here is the ratio of the product ion number vs. the input photon number and is different from "quantum yield", which is usually used in one-photon-photochemistry. 


\section{Discussion}

4.1. Reduction by $e_{\text {sol }}^{-}$as a major Au formation route and quenching of $e_{\text {sol }}^{-}$by dense $e_{\text {sol }}^{-}$and $\mathrm{OH} \bullet$ leading to a low efficiency

Irradiation of aqueous $\left[\mathrm{AuCl}_{4}^{-}\right]$solution using short and focused laser pulses would include the following reactions as the primary processes. The most significant $\mathrm{Au}^{0}$ formation reaction will be shown reduction by $\mathrm{e}_{\mathrm{sol}}^{-}$in (5).

$$
\begin{aligned}
& \mathrm{H}_{2} \mathrm{O}+n h v \rightarrow \mathrm{H}_{2} \mathrm{O}^{+}+\mathrm{e}^{-} \rightarrow \mathrm{H}_{2} \mathrm{O}^{+}+\mathrm{e}_{\mathrm{sol}}{ }^{-} \rightleftharpoons \mathrm{H}_{2} \mathrm{O} \\
& \mathrm{H}_{2} \mathrm{O}+n h v \rightarrow \mathrm{OH} \bullet+\mathrm{H} \bullet \rightleftharpoons \mathrm{H}_{2} \mathrm{O} \\
& {\left[\mathrm{AuCl}_{4}^{-}\right]+n h v \rightarrow \mathrm{Au}^{0}+3 \mathrm{Cl} \bullet+\mathrm{Cl}^{-}} \\
& {\left[\mathrm{AuCl}_{4}^{-}\right]+3 \mathrm{H} \bullet \rightarrow \mathrm{Au}^{0}+3 \mathrm{HCl}^{-} \mathrm{Cl}^{-}} \\
& {\left[\mathrm{AuCl}_{4}^{-}\right]+3 \mathrm{e}_{\mathrm{sol}}^{-} \rightarrow \mathrm{Au}^{0}+4 \mathrm{Cl}^{-}} \\
& \mathrm{H} \bullet^{-}+\mathrm{H}_{2} \mathrm{O} \rightarrow \mathrm{H}_{3} \mathrm{O}^{+}+\mathrm{e}_{\mathrm{sol}}^{-} \\
& \mathrm{e}_{\mathrm{sol}}^{-}+\mathrm{e}_{\mathrm{sol}}^{-} \rightarrow \mathrm{H}_{2}+\mathrm{OH}^{-} \\
& \mathrm{e}_{\mathrm{sol}}{ }^{-}+\mathrm{OH}^{-} \rightarrow \mathrm{OH}^{-} \\
& \mathrm{e}_{\mathrm{sol}}{ }^{-}+\mathrm{O}_{2} \rightarrow \mathrm{O}_{2}^{-}
\end{aligned}
$$

The five Reactions (1)-(5) are quoted from [8] and Reaction (6) between $\mathrm{H} \bullet$ and $\mathrm{H}_{2} \mathrm{O}$ is anotherformation $\mathrm{e}_{\mathrm{sol}}$ - process, which has been proposed in experiments of multiphoton ionization of water.[30] Intense laser pulses with a short duration can ionize and dissociate water molecules by multiphoton excitation, ionization, and dissociation of water and give hydrated electrons $\left(\mathrm{e}_{\mathrm{aq}}{ }^{-}\right)$, hydronium cations and hydroxyl radicals through 
Reaction (1), (2), and (6),[30-32] followed by the reduction by electrons to Au atom in (5). The $\mathrm{Au}^{0}$ formation reaction (4) would be unlikely, because the rate of $\mathrm{H} \bullet$ reactions in (6) will be so high that the diffusion reaction between $\left[\mathrm{AuCl}_{4}{ }^{-}\right]$and $\mathrm{H} \cdot$ would not overcome the $\mathrm{H} \cdot$ quenching reaction (6). The direct atomization by multi-photon absorption in (3) was not suggested to be a major mechanism.[8] In fact, there were no signs of atomization Reaction (3) based on the absorption experiments in section 3.1 of Results, because there was no enhanced absorption by $\mathrm{AuCl}_{4}{ }^{-}$. Above all, the major $\mathrm{Au}^{0}$ would be formed with the $\mathrm{e}_{\text {sol }}{ }^{-}$reduction in the reaction (5).

To discuss the low reduction efficiencies, three quenching reactions of $\mathrm{e}_{\mathrm{sol}}^{-}$(7), (8), and (9) are added. The weak plasma states are created in the present experiments in a very small volume; in other words, the concentrations of electrons and radicals immediately after excitation are so high that a rapid electron annihilation (7) and reaction with $\mathrm{OH} \bullet$ (8) presumably occur.

The formation yield of $\mathrm{e}_{\text {sol }}{ }^{-}$is 0.11 at $282 \mathrm{~nm}$ multiphoton excitation.[32] After the ionization water, several relaxation stages and recombination processes are required for the formation of $\mathrm{e}_{\text {sol }}{ }^{-}$in Reaction (1).[30-33] Since the generation of SC is an event within the excitation laser pulse, the relaxation and recombination stages occur after the SC generation. Two relaxation times (180 and $540 \mathrm{fs}$ ) and the recombination to $\mathrm{H}_{2} \mathrm{O}$, the so-called geminate recombination (tens picoseconds), have been reported.[30-33] The hydrated electrons react with $\mathrm{AuCl}_{4}{ }^{-}$with a high reaction rate constant of $6.1 \times 10^{10} \mathrm{M}^{-1} \mathrm{~s}^{-}$ ${ }^{1}$,[34] which can be classified as a diffusion controlled reaction, $k_{\text {diff. [35] The diffusion }}$ events occur in a few tens nanosecond scale $\left(\tau_{\mathrm{r}}\right)$ for the case of the $0.5 \mathrm{mM}$ Au complex ion system $\left(\tau_{\mathrm{r}=} k_{\text {diff }} \times\left[\mathrm{AuCl}_{4}^{-}\right]=3.1 \times 10^{7} \mathrm{~s}^{-1}\right.$ or the reaction time of $\left.32 \mathrm{~ns}\right)$.

The $\mathrm{e}_{\mathrm{sol}}{ }^{-}+\mathrm{e}_{\mathrm{sol}}{ }^{-}$annihilation in (7) is a second-order reaction and is expected to occur 
in several tens nanoseconds. We adopted the electron density (electrons before relaxation stages) as $2 \times 10^{19} \mathrm{~cm}^{-3}$ from Milián's analysis.[15] Assuming that $11 \%$ of the dry electrons survive as $\mathrm{e}_{\mathrm{sol}}{ }^{-}$, we estimated the concentration of $\left[\mathrm{e}_{\mathrm{sol}}{ }^{-}\right] \sim 3 \times 10^{-3} \mathrm{M}$. The initial $\mathrm{e}_{\mathrm{sol}}{ }^{-}$could decrease to half in about $60 \mathrm{~ns}$ using the bimolecular rate constant of $5.5 \times 10^{9} \mathrm{M}^{-1} \mathrm{~s}^{-1}$.[35] The reaction between $\mathrm{e}_{\mathrm{sol}}{ }^{-}$and $\mathrm{OH} \bullet$ in (8) would more efficiently quench $\mathrm{e}_{\mathrm{sol}}{ }^{-}$than that the annihilation (7). In addition to a high reaction rate constant in (8), a high concentration of the $\mathrm{OH} \bullet$ radical is expected under the present conditions. The $\mathrm{OH} \bullet$ radical in (8) will be generated through (2), after the initial dissociation with a yield of 0.74 [31] and recombination. The concentration of the $\mathrm{OH} \bullet$ radical could be $10^{-2} \mathrm{M}$, and then the quenching time using the reaction rate in (8) of $3.0 \times 10^{10} \mathrm{M}^{-1} \mathrm{~s}^{-1}$ [35] would be $3 \mathrm{~ns}$; this is about 10 times more efficient than the reaction of $\mathrm{e}_{\text {sol }}{ }^{-}$and $\mathrm{AuCl}_{4}{ }^{-}$in (5). These two quenching reactions (7) and (8) would be formidable competing reactions to form $\mathrm{Au}^{0}$ with the reaction (5). Reaction (9) is a lifetime-determination-process of $\mathrm{e}_{\mathrm{sol}}{ }^{-}$in air saturated water. It is a slow process and can be neglected, but dissolved oxygen would quench $\mathrm{e}_{\text {sol }}^{-}$by $\mathrm{e}_{\mathrm{sol}}{ }^{-}+\mathrm{O}_{2} \rightarrow \mathrm{O}_{2}^{-}$with $1.9 \times 10^{10} \mathrm{M}^{-1} \mathrm{~s}^{-1}$,[12] which is an event occurring in $200 \mathrm{~ns}$, based on $\left[\mathrm{O}_{2}\right] \sim 2.8 \times 10^{-4} \mathrm{M}$ in an air saturated solution.

On the basis of the above considerations, an efficiency of $10^{-3}$ is expected for the femtosecond reductions. The efficiency $(\eta)$ is proportional to (absorbed photons $) \times(5$ photon excitation $) \times\left(\mathrm{e}_{\text {sol }}{ }^{-}\right.$yield $) \times\left(\mathrm{e}_{\text {sol }}{ }^{-}\right.$survival efficiency overcoming (7) and (8) ) and $\times\left(\right.$ reaction (5)). Adopting the actually absorbed pulse energy of $15 \%$ and $11 \%$ of $^{\mathrm{sol}^{-}}$ yield,[32] under the assumption that all absorbed energy is used to excite or ionize water molecules, $\eta$ should be 0.15 (absorbed photons) $\times 1 / 5(5$-photon abs. $) \times 0.11\left(\mathrm{e}_{\mathrm{sol}}{ }^{-}\right.$ yield $) \times\left(\mathrm{e}_{\text {sol }}{ }^{-}\right.$survival $) \times($reaction $(5))=3.7 \times 10^{-3} \times\left(\mathrm{e}_{\text {sol }}{ }^{-}\right.$survival $) \times($reaction $(5))$. The survival efficiency of $\mathrm{e}_{\mathrm{sol}}{ }^{-}$and the followed by reaction (5) could reach the observed order 
$\eta$ of $10^{-3}$. In conclusion, the conditions under focused femtosecond pulses create high concentrations of $\mathrm{e}_{\mathrm{sol}}^{-}$and $\mathrm{OH} \bullet$ radical. The annihilation reaction (7) and the quenching reaction by $\mathrm{OH} \bullet(8)$ would give an efficiency on the order of $10^{-3}$. The above discussion would be applicable for the ions treated by the authors, $\mathrm{Eu}^{3+}, \mathrm{Yb}^{3+}, \mathrm{Sm}^{3+}, \mathrm{Ag}^{+}, \mathrm{Pd}^{2+}$, and $\mathrm{Fe}\left(\mathrm{C}_{2} \mathrm{O}_{4}\right)_{3}{ }^{3-}$. Reactions of metal ions with $\mathrm{e}_{\mathrm{aq}}{ }^{-}$have been commonly involved with diffusion controlled rate constants.[35-37] 


\subsection{Reasonable multiphoton absorption cross sections}

It will be shown that the ratio of multiphoton absorption cross sections $\sigma^{(n)} / \sigma^{(n-1)}$ are in a reasonable range. The values $\sigma^{(n)} / \sigma^{(n-1)}$ are $10^{-34}$ for a general case [38] and $10^{-32}$ for 2-4 photon absorption in $p$-terphenyl.[39] The following two relations are adopted for the discussion of $\sigma^{(n)} / \sigma^{(n-1)}$ based on our observations.

$$
\begin{aligned}
& \sigma^{(5)} I^{4}\left[\mathrm{H}_{2} \mathrm{O}\right]>\sigma^{(3)} I^{2}\left[\mathrm{AuCl}_{4}{ }^{-}\right] \\
& \sigma^{(5)} I^{4}\left[\mathrm{H}_{2} \mathrm{O}\right] \geq \sigma^{(2)} I\left[\mathrm{PdCl}_{2}\right]
\end{aligned}
$$

where $\sigma^{(n)}$ is an $n$ th-order multiphoton absorption cross section, $I$ is a laser intensity, and [M-complex] is a solute concentration. The absorption measurements described in section 3.1 of Results indicate that water plays a major role under focused conditions. The solutions here contain $\mathrm{Ag}+, \mathrm{AuCl}_{4}{ }^{-}, \mathrm{SDS}, \mathrm{M}^{\mathrm{n}+}\left(\mathrm{H}_{2} \mathrm{O}\right)_{\mathrm{m}}, \mathrm{X}^{-}$, where $\mathrm{M}$ is metal, and $\mathrm{X}$ is $\mathrm{ClO}_{4}$ and $\mathrm{Cl}$, and are practically transparent at the laser wavelength of $800 \mathrm{~nm}$. They did not show extra absorption at the concentrations used here. The laser intensity $I$ is adopted to be $2 \times 10^{13} \mathrm{Wcm}^{-2}$ by comparing the present water absorption with the values reported in the literature.[15] Under self-focusing conditions, a simple uniform focusing method can no longer be applied for the evaluation of laser intensity. Self-focusing conditions induce pulse shape changing and intensity clamping; therefore, the laser intensity reaches and/or cannot exceed this value, where the non-linear absorption is expected. A mechanism of multi-photon absorption of water is adopted here, though tunneling ionization may have started.[15]

Inequality (10) reflects the following observations. Water absorbed at about $15 \%$ 
of input laser energy and five-photon absorption are required to overcome the water ionization potential of $6.5 \mathrm{eV}$.[40] Three-photon absorption can reach an excited state of $\mathrm{AuCl}_{4}{ }^{-}$, but its solution did not show an extra decrease in laser energy. Inequality (11) indicates that there is $3 \%$ extra absorption in $\mathrm{PdCl}_{2}$ solution vs. $15 \%$ extra absorption by water. Inequality (10) gives $\sigma^{(5)} / \sigma^{(3)}>10^{-69}$; vs. the predicted values of $\sigma^{(5)} / \sigma^{(3)}$ are $10^{-64}$, [39] $10^{-68}$ [38] from the extension of $\sigma^{(n)} / \sigma^{(n-1)}$ in the literature. In (11) $\sigma^{(5)} / \sigma^{(2)} \geq 10^{-99}$ vs. the predictions of $10^{-96},[39] 10^{-102}$.[38] The ratios of cross section values are close to the order of predicted values.

\subsection{Extra absorption by Fe and Pd systems, but not by SDS}

Extra absorptions were seen for the yellow colored solutions of Pd and Fe salts; the extra absorption was $3 \%$ for the Pd sample and about $10 \%$ for the Fe system, when water absorbs laser energy of $15 \%$ as described in section 3.1 of Results. For the case of the Fe complex, the additional absorptions are assigned to two-photon absorptions and the excited state of the Fe complex is photochemically active as discussed.[3] The $\mathrm{PdCl}_{2}$ solution has a d-d transition of $\mathrm{Pd}^{2+}$ around $400 \mathrm{~nm}$ as seen in Fig. 4 and the additional absorptions would be due to two-photon absorption to this absorption; however, this band is known to be inactive for NP formation by one-photon direct photo-excitation.[25]

SDS $\left(10^{-2} \mathrm{M}\right)$ was used for the Ag and Au NPs experiments but not for the Pd system. The absorption peak and intensities of NPs were kept almost constant for more than several hours with SDS. Though Ag and Au NPs were observed without SDS, their spectral reproducibility was poor. The SDS might have been ionized to some extent by the femtosecond laser pulses, but the contribution to the metal ion reduction would be 
much smaller than those of water itself. No additional absorption was observed in SDS $\left(10^{-2} \mathrm{M}\right)$ solution and in $\mathrm{Ag}$ and $\mathrm{Au}$ salt solutions with SDS, in the focused beam experiments described in section 3.1 of Results. It may be notable that Au NPs were synthesized at a low intensity femtosecond laser pulse and the formation was suggested to be mediated by four-photon ionization of a surfactant chitosan.[7]

\subsection{Comments on the slope of $n$ and SC at a low intensity}

The slopes of 2-5 for the Ag and Au NPs were observed and are presented in Fig. 3. Although the results suggested that we were observing nonlinear processes at least, these slopes cannot be definitively defined as $\mathrm{s} n$-th processes. The slope of $n$ for the $n$ order process is meaningful when the laser beam is geometrically focused without any deformation either geometrically and temporally. On the basis of water absorption and SC generation, the laser intensity at $3 \mu \mathrm{J}$ with a duration of $90 \mathrm{fs}$ was estimated to be $2 \times$ $10^{13} \mathrm{Wcm}^{-2}$, where intensity clamping and re-focusing of the laser pulse were going on. The slope of $n$ for the $n$-order multiphoton processes as a function of the laser energy tends to give a value lower than the real $n$-th order processes in a high intensity laser energy. Characteristic changes in the slope are observed for the case of $\mathrm{N}_{2}$ ionization experiments.[41] Similar changes even in the photochemistry of organic molecules in solution were observed, where the turning intensity was estimated to be $1 \times 10^{13} \mathrm{Wcm}^{-}$ ${ }^{2}$.[42] We have previously observed a slope of 1.5-1.9 for reduction of metal ions by a femtosecond laser.[3] Those experiments were done under laser intensities accompanied by SC generation. Unfortunately, the observed $n$-th slopes cannot cut into the present mechanisms. 


\section{Conclusions}

Metal ions in solution can be reduced finally reaching NPs for $\mathrm{Ag}, \mathrm{Au}$, and Pd ion systems, when femtosecond laser pulses are focused to an intensity level being accompanied by supercontinuum (SC) generation. The formation of NPs was observed by pulses with micro-Joule energy, which to our knowledge is the lowest energy yet used for this purpose. That was just around a threshold of SC generation and well below a condition accompanied by the strong optical breakdown (SOB). To understand the metal ion reduction mechanisms, the following points in the Introduction have been made clear.

i) The major absorbing species is water in transparent samples including $\mathrm{AuCl}_{4}{ }^{-}$ solution. The absorption amount reached $15 \%$ under focused femtosecond laser pulse conditions, where stable SC generation is seen and strong optical breakdown is not seen. The absence of multiphoton absorption indicates that $\mathrm{Ag} \mathrm{Au}$ and Pd reduction leading to NPs were observed without atomization of metal ionic species and/or surfactant (SDS) ionization.

ii) Consumption efficiencies $(\eta)$ of metal ion from incident photons for $\mathrm{Au}$ and $\mathrm{Pd}$ ion systems and previous studies of conversion efficiencies for $\mathrm{Yb}$ and Fe systems are on the order of $10^{-3}$ by focused micro-Joule femtosecond pulses.

iii) We eventually found rather low efficiencies. The competing quenching reactions were suggested to be electron-electron and electron- $\mathrm{OH} \bullet$ reactions. These quenching reactions would be caused by special conditions; the reductions would occur in a small volume by focused femtosecond laser pulses, which generates weak plasma states in a small volume. High concentrations of electrons and $\mathrm{OH} \bullet$ radicals co-exist. 


\section{Acknowledgements}

The authors thank Dr. Noritomo Suzuki (Toyota Central R\&D Labs. Inc.) for taking TEM images. This work was financially supported in part by a Grant-in-Aid (No. 23550030) from the Ministry of Education, Culture, Sports, Science, and Technology of Japan to N.N. 


\section{References}

[1] D. Nishida, M. Kusaba, T. Yatsuhashi, N. Nakashima, Reduction of $\mathrm{Eu}^{3+}$ to $\mathrm{Eu}^{2+}$ by an intense femtosecond laser pulse in solution, Chem. Phys. Lett. 465 (2008) 238-240.

[2] D. Nishida, E. Yamade, M. Kusaba, T. Yatsuhashi, N. Nakashima, Reduction of Sm ${ }^{3+}$ to $\mathrm{Sm}^{2+}$ by an intense femtosecond laser pulse in solution, J. Phys. Chem. A 114 (2010) 5648-5654.

[3] N. Nakashima, K. Yamanaka, T. Yatsuhashi, Ionic valence change of metal ions in solution by femtosecond laser excitation accompanied by white-light laser, Chin. J. Phys. 52 (2014) 504-518.

[4] C. Zhao, S. Qu, J. Qiu, C. Zhu, Photoinduced formation of colloidal Au by a nearinfrared femtosecond laser, J. Materials Res. 18 (2003) 1710-1714.

[5] S. Besner, M. Meunier, Femtosecond laser synthesis of AuAg nanoalloys: photoinduced oxidation and ions release, J. Phys. Chem. C 114 (2010) 10403-10409.

[6] T. Nakamura, Y. Herbani, D. Ursescu, R. Banici, R. V. Dabu, S. Sato, Spectroscopic study of gold nanoparticle formation through high intensity laser irradiation of solution, AIP Advances 3 (2013) 082101 (6 pages).

[7] P. H. D. Ferreira, M. G. Vivas, L. De Boni, D. S. dos Santos Jr., D. T. Balogh, L. Misoguti, C. R. Mendonca, Femtosecond laser induced synthesis of Au nanoparticles mediated by chitosan, Opt. Express 20 (2012) 518-523.

[8] B. Tangeysh K. M. Tibbetts, J. H. Odhner, B. B. Wayland, R. J. Levis, Gold nanoparticle synthesis using spatially and temporally shaped femtosecond laser pulses: post-irradiation auto-reduction of aqueous $\left[\mathrm{AuCl}_{4}\right]^{-}$, J. Phys. Chem. C 117 
(2013) 18719-18727.

[9] S. Besner, A. V. Kabashin, F. M. Winnik, M. Meunier, Synthesis of size-tunable polymer-protected gold nanoparticles by femtosecond laser-based ablation and seed growth. J. Phys. Chem. C 113(2009) 9526-9531.

[10] T. Nakamura, H. Magara, Y. Herbani, S. Sato, Fabrication of silver nanoparticles by highly intense laser irradiation of aqueous solution, Appl. Phys. A 104 (2011) 10211024.

[11] E. Akman, B. G. Oztoprak, M. Gunes, E. Kacar, A. Demir, Effect of femtosecond Ti:Sapphire laser wavelengths on plasmonic behaviour and size evolution of silver nanoparticles, Photonics and Nanostructures - Fundamentals and Applications, 9 (2011) 276-286.

[12] G. Fan, S. Ren, S. Qu, Z. Guo, Q. Wang, Y. Wang, R. Gao, Mechanisms for fabrications and nonlinear optical properties of $\mathrm{Pd}$ and $\mathrm{Pt}$ nanoparticles by femtosecond laser, Opt. Commun. 295 (2013) 219-225.

[13] G. Fan, S. Qu, Q. Wang, C. Zhao, L. Zhang, Z. Li, Pd nanoparticles formation by femtosecond laser irradiation and the nonlinear optical properties at $532 \mathrm{~nm}$ using nanosecond laser pulses, J. Appl. Phys. 109 (2011) 023102 (7 pages).

[14] D. Tan, Z. Shifeng, J. Qiu, N. Khusro, Preparation of functional nanomaterials with femtosecond laser ablation in solution, J. Photochem. Photobiolo. C: Photochem. Rev. 17 (2013) 50-68.

[15] C. Milián, A. Jarnac, Y. Brelet, V. Jukna, A. Houard, A. Mysyrowicz, A. Couairon, Effect of input pulse chirp on nonlinear energy deposition and plasma excitation in water, J. Opt. Soc. Am. B 31(2014) 2829-2837.

[16] W. Liu, S. Petit, A. Becker, N. Aközbek, C.M. Bowden, S.L. Chin, Intensity 
clamping of a femtosecond laser pulse in condensed matter, Optics Communications 202 (2002) 189-197.

[17] T. Yatsuhashi, N. Uchida, K. Nishikawa, Novel method of producing carbon nanoparticles on benzene/water interface with femtosecond laser plasma filament, Chem. Lett. 41(2012) 722-724.

[18] K. Yamanaka, T. Morikawa, Charge-carrier dynamics in nitrogen-doped $\mathrm{TiO}_{2}$ powder Studied by femtosecond time-resolved diffuse reflectance spectroscopy, J. Phys. Chem. C 116(2012) 1286-1292.

[19] D. Kosumi, T. Nishiguchi, M. Sugisaki, H. Hashimoto, Ultrafast coherent spectroscopic investigation on photosynthetic pigment chlorophyll a utilizing 20 fs pulses, J. Photochem. Photobiol. A: Chem. X (2015) xxx-xxx.

[20] U. Y. Qazi, S. Kajimoto, H. Fukumura, Effect of sodium dodecyl sulfate on the formation of silver nanoparticles by biphotonic reduction of silver nitrate in water, Chem. Lett. 43 (2014) 1693-1695.

[21] C. Petit, P. Lixon, M. P. Pileni, In situ synthesis of silver nanocluster in AOT reverse micelles, J. Phys. Chem. 97 (1993) 12974-12983.

[22] A. Henglein, Physicochemical properties of small metal particles in solution: "microelectrode" reactions, chemisorption, composite metal particles, and the atom-to-metal transition, J. Phys. Chem. 97 (1993) 5457-5471.

[23] F. Mafuné, J. Kohno, Y. Takeda, T. Kondow, H. Sawabe, Structure and stability of silver nanoparticles in aqueous solution produced by laser ablation. J. Phys. Chem. B 104 (2000) 8333-8337. 
[24] F. Mafuné, J. Kohno, Y. Takeda, T. Kondow, H. Sawabe, Formation of gold nanoparticles by laser ablation in aqueous solution of surfactant, J. Phys. Chem. B 105 (2001) 5114-5120.

[25] M. Saeki, T. Taguchi, N. Nakashima, H. Ohba, Wet separation between Palladium(II) and Molybdenum(IV) ions by using laser-induced particle formation: enhancement of recovery efficiency of palladium by laser condition, J. Photochem. Photobiol. A 299 (2014) 189-193.

[26] E. Ye, H. Tan, S. Li, W. Y. Fan, Self-organization of spherical, core-shell palladium aggregates by laser-induced and thermal decomposition of $\left[\mathrm{Pd}\left(\mathrm{PPh}_{3}\right)_{4}\right]^{\dagger}$. Angew. Chem. Int. Ed. 45 (2006) 1120-1123.

[27] H. Tan, T. Zhan, W. Y. Fan, A simple route to water-soluble size-tunable monodispersed $\mathrm{Pd}$ nanoparticles from light decomposition of $\mathrm{Pd}\left(\mathrm{PPh}_{3}\right)_{4}$. Chem. Phys. Lett. 428 (2006) 352-355.]

[28] Herbani, Y.; Nakamura, T.; Sato, S. Femtosecond laser-induced formation of goldrich nanoalloys from the aqueous mixture of gold-silver ions. J. Nanomaterials. 2010 (2010) 154210, 9 pages.

[29] W. Liu, O. Kosareva, I. S. Golubtsov, A. Iwasaki, A. Becker, V .P. Kandido, S. L. Chin, Femtosecond laser pulse filamentation versus optical breakdown in $\mathrm{H}_{2} \mathrm{O}$, Appl. Phys. B 76(2003) 215-229.

[30] R. A. Crowell, D. M. Bartels, Multiphoton ionization of liquid water with 3.0-5.0 eV photons, J. Phys. Chem. 100 (1996) 17940-17949.

[31] S. Pommeret, F. Gobert, M. Mostafavi, I. Lampre, J.-C. Mialocq, Femtochemistry of the hydrated electron at decimolar concentration, J. Phys. Chem. A 2001, 105 (2001) 11400-11406. 
[32] A. Reuther, A. Laubereau, D. N. Nikogosyan, Primary Photochemical Processes in Water, J. Phys. Chem. 100 (1996) 16794-16800

[33] M. Mizuno, S. Yamaguchi, T. Tahara, Relaxation dynamics of the hydrated electron: femtosecond time-resolved resonance Raman and luminescence study, J. Phys. Chem. A 109 (2005) 5257-5265.

[34] D. Behar, J. Rabani, Kinetics of hydrogen production upon reduction of aqueous $\mathrm{TiO} 2$ nanoparticles catalyzed by $\mathrm{Pd}_{0}, \mathrm{Pt}_{0}$, or $\mathrm{Au}_{0}$ coatings and an unusual hydrogen abstraction; steady state and pulse radiolysis study, J. Phys. Chem. B 110 (2006) $8750-8755$.

[35] G. V. Buxton, C. L. Greenstock, W. P. Helman, A. B. Ross, Critical review of rate constants for reactions of hydrated electrons, hydrogen atoms and hydroxyl radicals $\left(\bullet \mathrm{OH} / \bullet \mathrm{O}^{-}\right)$in aqueous solution, J. Phys. Chem. Ref. Data 17 (1988) 513-886.

[36] M. Michaelis, A. Henglein, Reduction of Pd(I1) in aqueous solution: stabilization and reactions of an intermediate cluster and Pd colloid formation, J. Phys. Chem. 96(1992) 4719-4724.

[37] J. Chen, H. Zhang, I. V. Tomov, P. M. Rentzepis, Electron transfer mechanism and photochemistry of ferrioxalate induced by excitation in the charge transfer band, Inorg. Chem. 47 (2008) 2024-2032.

[38] Y.R. Shen, Multiphoton Spectroscopy, Chap.18 in The principles of nonlinear optics, New York, J. Wiley, 1984, pp334-349.

[39] T. Yatsuhashi, S. Ichikawa, Y. Shigematsu, N. Nakashima, High-order multiphoton fluorescence of organic molecules in solution by intense femtosecond laser pulses, J. Am. Chem. Soc. 130 (2008) 15264-15265.

[40] D. N. Nikogosyan, A. A. Oraevsky, V. I. Rupasov, Two-photon ionization and 
dissociation of liquid water by powerful laser UV radiation, Chem. Phys. 77, 131(1983).

[41] A. Becker, N. Aközbek, K. Vijayalakshmi, E. Oral, C. M. Bowden, S. L. Chin, Intensity clamping and re-focusing of intense femtosecond laser pulses in nitrogen molecular gas, Appl. Phys. B 73 (2001) 287-290.

[42] T. Yatsuhashi, Y. Nakahagi, H. Okamoto, N. Nakashima, Linear response of multiphoton reaction: three-photon cycloreversion of anthracene biplanemer in solution by intense femtosecond laser pulses, J. Phys. Chem. A 114(2010) 1047510480. 